\title{
A NUMERICAL METHOD FOR THE MODIFIED VECTOR-VALUED ALLEN-CAHN PHASE-FIELD MODEL AND ITS APPLICATION TO MULTIPHASE IMAGE SEGMENTATION
}

\author{
HYUN GEUN LEE ${ }^{1}$ AND JUNE-YUB LEE ${ }^{2}$ \\ ${ }^{1}$ Institute of Mathematical Sciences, Ewha Womans University, SeOUl 120-750, Korea \\ E-mail address: leeh1 dewha.ac.kr \\ ${ }^{2}$ Department of Mathematics, Ewha Womans University, Seoul 120-750, Korea \\ E-mail address: jyllee@ewha.ac.kr
}

\begin{abstract}
In this paper, we present an efficient numerical method for multiphase image segmentation using a multiphase-field model. The method combines the vector-valued AllenCahn phase-field equation with initial data fitting terms containing prescribed interface width and fidelity constants. An efficient numerical solution is achieved using the recently developed hybrid operator splitting method for the vector-valued Allen-Cahn phase-field equation. We split the modified vector-valued Allen-Cahn equation into a nonlinear equation and a linear diffusion equation with a source term. The linear diffusion equation is discretized using an implicit scheme and the resulting implicit discrete system of equations is solved by a multigrid method. The nonlinear equation is solved semi-analytically using a closed-form solution. And by treating the source term of the linear diffusion equation explicitly, we solve the modified vector-valued Allen-Cahn equation in a decoupled way. By decoupling the governing equation, we can speed up the segmentation process with multiple phases. We perform some characteristic numerical experiments for multiphase image segmentation.
\end{abstract}

\section{INTRODUCTION}

The Allen-Cahn (AC) equation [1] was originally introduced as a phenomenological model for antiphase domain coarsening in a binary alloy. The AC equation and its modified forms have been widely adapted to model problems such as phase transitions [1], crystal growth [2, 3, 4, 5], grain growth $[6,7,8,9,10]$, image segmentation $[11,12]$, motion by mean curvature $[13,14$, $15,16,17,18,19]$, two-phase fluid flows [20], and vesicle membranes [21]. To solve the AC equation and its modified forms numerically, various methods have been developed including boundary integral [22, 23], cellular automata [24, 25], front-tracking [26, 27], level-set [28, 29,

Received by the editors October 31 2013; Accepted November 15 2013; Published online February 242014.

2000 Mathematics Subject Classification. 65M06.

Key words and phrases. Modified vector-valued Allen-Cahn equation, Multiphase-field model, Multiphase image segmentation, Operator splitting method, Multigrid.

This research was supported by Basic Science Research Program through the National Research Foundation of Korea(NRF) funded by the Ministry of Education(2009-0093827, 2012-002298). 
30], and phase-field [30, 31, 32, 33, 34, 35] methods. Among these various methods, the phasefield method is popular and widely used. The most significant computational advantage of the phase-field method is that an explicit tracking of the interface is unnecessary by introducing a phase-field variable.

An important class of phase-field models are multiphase-field models [36, 37, 38, 39, 40, $41,42,43,44]$ which represent a multiphase microstructure by a vector-valued phase-field $\phi$ with $K$ components $\left(\phi_{1}, \ldots, \phi_{K}\right)$. Each component is called a phase-field variable and is continuous in space and time. Inside a bulk phase, one component $\phi_{k}$ takes the value one, while the other phase-field variables assume the value zero. In accordance with [36], the condition is imposed that the components $\phi_{k}$ must lie in the Gibbs $K$-simplex

$$
G:=\left\{\phi \in \mathbb{R}^{K} \mid \phi_{k} \geq 0, \sum_{k=1}^{K} \phi_{k}=1\right\} .
$$

Without loss of generality, we postulate that the Ginzburg-Landau total free energy of the multiphase microstructure can be written as follows:

$$
\mathcal{E}(\phi)=\int_{\Omega}\left(\frac{F(\phi)}{\epsilon^{2}}+\frac{1}{2} \sum_{k=1}^{K}\left|\nabla \phi_{k}\right|^{2}\right) d \mathbf{x},
$$

where $\Omega \subset \mathbb{R}^{d}(d=1,2,3)$ is a polygonal (polyhedral) domain, $F(\phi)=0.25 \sum_{k=1}^{K} \phi_{k}^{2}(1-$ $\left.\phi_{k}\right)^{2}$, and $\epsilon>0$ is the gradient energy coefficient. The vector-valued AC equation [36, 38, 40, $41,42,43,44]$ is the $L^{2}$-gradient flow of the total free energy $\mathcal{E}(\phi)$ defined in (1.2) under the additional constraint (1.1), which has to hold everywhere at any time. It is natural to seek a law of evolution in the form

$$
\frac{\partial \phi}{\partial t}=-\operatorname{grad} \mathcal{E}(\phi)
$$

The symbol "grad" here denotes the gradient on the manifold in $L^{2}$ space. Let $\mathbf{f}(\phi)=$ $\left(f\left(\phi_{1}\right), \ldots, f\left(\phi_{K}\right)\right)$ be the gradient of $F, \frac{\partial F}{\partial \phi}=\left(\frac{\partial F}{\partial \phi_{1}}, \ldots, \frac{\partial F}{\partial \phi_{K}}\right)$ where $f(\phi)=\phi(\phi-$ $0.5)(\phi-1)$ and we use a variable Lagrange multiplier $\beta(\phi)=(-1 / K) \sum_{k=1}^{K} f\left(\phi_{k}\right)$ [36] in order to ensure the constraint (1.1). Using a general smooth vector-valued function $\boldsymbol{\xi}$, we set

$$
\boldsymbol{\psi}=\left(\psi_{1}, \ldots, \psi_{K}\right)=\boldsymbol{\xi}-\frac{1}{K} \sum_{k=1}^{K} \xi_{k} \mathbf{1},
$$

where $\mathbf{1}=(1, \ldots, 1) \in \mathbb{R}^{K}$ and $\sum_{k=1}^{K} \psi_{k}=0$. Then we have

$$
\begin{aligned}
&(\operatorname{grad}\mathcal{E}(\boldsymbol{\phi}), \boldsymbol{\psi})_{L^{2}}=\left.\frac{d}{d \theta} \mathcal{E}(\boldsymbol{\phi}+\theta \boldsymbol{\psi})\right|_{\theta=0} \\
& \quad=\int_{\Omega}\left[\frac{\mathbf{f}(\boldsymbol{\phi})+\beta(\boldsymbol{\phi}) \mathbf{1}}{\epsilon^{2}}-\Delta \boldsymbol{\phi}\right] \cdot \boldsymbol{\psi} d \mathbf{x}=\left(\frac{\mathbf{f}(\boldsymbol{\phi})+\beta(\boldsymbol{\phi}) \mathbf{1}}{\epsilon^{2}}-\Delta \boldsymbol{\phi}, \boldsymbol{\psi}\right)_{L^{2}},
\end{aligned}
$$


where we have used the zero Neumann boundary condition: $\nabla \phi_{k} \cdot \mathbf{n}=0$ on $\partial \Omega$, where $\mathbf{n}$ is the unit normal vector to $\partial \Omega$. We identify $\operatorname{grad} \mathcal{E}(\phi) \equiv(\mathbf{f}(\boldsymbol{\phi})+\beta(\boldsymbol{\phi}) \mathbf{1}) / \epsilon^{2}-\Delta \boldsymbol{\phi}$, then $(1.3)$ becomes the vector-valued $\mathrm{AC}$ equation

$$
\frac{\partial \phi}{\partial t}=-\frac{\mathbf{f}(\phi)+\beta(\phi) \mathbf{1}}{\epsilon^{2}}+\Delta \phi .
$$

Until recent years, only explicit discretization schemes have been used to solve the multiphasefield model numerically [36, 38, 39]. Unfortunately, these discretization methods suffer from a severe time step restriction. To avoid the strong stability condition of traditional explicit methods, Vanherpe et al. [43] employed a semi-implicit discretization scheme that treats the gradient energy part of the model implicitly and the potential part explicitly. And Lee and Kim [44] presented a hybrid operator splitting method for solving the model, in which the linear diffusion part of the model was solved using an implicit scheme and the nonlinear part was solved semi-analytically.

In this paper, we extend the vector-valued AC model (1.4) into a modified vector-valued AC model with initial data fitting terms containing prescribed interface width and fidelity constants to solve multiphase image segmentation problem [35, 45, 46, 47, 48]. The objective of this paper is to present an efficient numerical method for multiphase image segmentation. We employ the recently developed hybrid operator splitting method for the vector-valued AC model [44]. We split the modified vector-valued AC model into a nonlinear part and a linear diffusion part with a source term (the initial data fitting terms and the constraint terms). The linear diffusion part is discretized using an implicit scheme and the resulting implicit discrete system of equations is solved by a fast solver, such as a multigrid method $[49,50]$. The nonlinear part is solved semi-analytically using a closed-form solution. And by treating the source term of the linear diffusion part explicitly, we solve the modified vector-valued AC model in a decoupled way, i.e., we only solve the modified scalar AC equation $K-1$ times to solve the modified vector-valued AC equation since $\phi_{K}=1-\sum_{k=1}^{K-1} \phi_{k}$. By decoupling the governing equation, we can speed up the segmentation process with multiple phases.

This paper is organized as follows. In Section 2, the proposed model for multiphase image segmentation is given. In Section 3, we describe an efficient numerical method which is based on an operator splitting method. In Section 4, we perform some characteristic numerical experiments for multiphase image segmentation. Finally, conclusions are given in Section 5.

\section{Modified ALLEN-CAHN PHASE-FIELD MODEL FOR IMAGE SEGMENTATION}

Image segmentation is one of the most important tasks in computer vision. Its goal is to partition a given image into several regions so that each region has uniform characteristics such as edges, intensities, color, and texture. Variational methods have been increasingly used as powerful methods for image segmentation. In variational formulation, image segmentation is achieved by solving an energy minimization problem. Many variational models for image segmentation have been proposed in the past two decades [51, 52, 53, 54]. One of the most successful models is the Mumford-Shah model [51], which approximates an image by a piecewise smooth function with regular boundaries: Given a grayscale image $I: \Omega \rightarrow \mathbb{R}$, where the 
image domain $\Omega$ is a bounded and open subset of $\mathbb{R}^{2}$ such that $\Omega=\bigcup_{i=1}^{K} \Omega_{i}\left(\Omega_{i}\right.$ are several subdomains with $\Omega_{i} \cap \Omega_{j}=\emptyset$ when $i \neq j$ ), the Mumford-Shah functional is given by

$$
\mathcal{E}_{M S}(\phi, \Gamma)=\int_{\Omega \backslash \Gamma}|\nabla \phi|^{2} d \mathbf{x}+\mu \int_{\Gamma} d \sigma+\lambda \int_{\Omega}(\phi-I)^{2} d \mathbf{x},
$$

where $\Gamma=\bigcup \partial \Omega_{i}$ is the union of boundaries of $\Omega_{i}, \phi$ is a piecewise smooth approximate function of image $I$, and $\mu, \lambda$ are positive constants. The first term minimizes the variation of $\phi$ and promotes its smoothness, the second term minimizes the length of interfaces and determines the boundaries between $\Omega_{i}$, and the third term, sometimes referred to as the fidelity or fitting term, minimizes the variation between $\phi$ and $I$. In particular, Mumford and Shah considered the special case where the function $\phi$ is chosen to be a piecewise constant function. The piecewise constant Mumford-Shah functional was rediscovered by Chan and Vese with a level set formulation [55].

A phase-field approximation for minimizing the piecewise constant Mumford-Shah functional is given by the following functional:

$$
\mathcal{E}(\phi)=\int_{\Omega}\left(\frac{F(\phi)}{\epsilon^{2}}+\frac{|\nabla \phi|^{2}}{2}+\frac{\lambda}{2}\left[\phi^{2}\left(I-c_{1}\right)^{2}+(1-\phi)^{2}\left(I-c_{2}\right)^{2}\right]\right) d \mathbf{x},
$$

where we have added two fitting terms to the Ginzburg-Landau energy, $F(\phi)=\phi^{2}(1-\phi)^{2}$ is a double well potential, and $c_{1}$ and $c_{2}$ are averages of $I$ in the regions in which $\phi$ is one and zero, respectively:

$$
c_{1}=\frac{\int_{\Omega} I \phi d \mathbf{x}}{\int_{\Omega} \phi d \mathbf{x}} \quad \text { and } \quad c_{2}=\frac{\int_{\Omega} I(1-\phi) d \mathbf{x}}{\int_{\Omega}(1-\phi) d \mathbf{x}} .
$$

Variation of energy (2.1) with respect to $\phi$ yields the following gradient descent equation (the modified scalar AC equation):

$$
\phi_{t}=-\frac{f(\phi)}{\epsilon^{2}}+\Delta \phi-\lambda\left[\phi\left(I-c_{1}\right)^{2}-(1-\phi)\left(I-c_{2}\right)^{2}\right] .
$$

Multiphase segmentation is a more challenging problem than two-phase segmentation. Main difficulty is in finding effective representations of the regions and their boundaries. Several recent works are related to the multiphase Mumford-Shah model [35, 46, 45, 47, 48]. Vese and Chan [45] generalized the two-phase model [55] to multiphase segmentation by using multiple level set functions. Both piecewise constant and piecewise smooth cases are studied. Their model can segment an image into $2^{K}$ phases with $K$ level-set functions. Thus, their model evolves more regions than necessary whenever the number of regions is not a power of two. In this case, the redundant regions are empty. Samson et al. [46] proposed another level set based multiphase segmentation model by adding a penalty term on the level set functions to penalize the vacuum and overlap. Lie et al. [47] introduced a piecewise constant level set function and used each constant value to represent a unique phase in a piecewise constant segmentation model. Jung et al. [48] proposed a phase field method to handle multiphase piecewise constant segmentation. The method is based on the phase transition model of Modica and Mortola with a sinusoidal potential. Li and Kim [35] modified the sinusoidal potential in the model of Jung et 
al. to a periodic quartic polynomial potential and presented an efficient and accurate numerical scheme by using the polynomial potential.

In this paper, to achieve a simultaneous segmentation of $I$ into arbitrarily many pieces, we refer to the vector-valued formulation of the AC equation. Similar to (2.1), we add initial data fitting terms to the Ginzburg-Landau energy (1.2) and then obtain

$$
\mathcal{E}(\phi)=\int_{\Omega}\left(\frac{F(\phi)}{\epsilon^{2}}+\frac{1}{2} \sum_{k=1}^{K}\left|\nabla \phi_{k}\right|^{2}+\frac{\lambda}{2} \sum_{k=1}^{K} \phi_{k}^{2}\left(I-c_{k}\right)^{2}\right) d \mathbf{x},
$$

where

$$
c_{k}=\frac{\int_{\Omega} I \phi_{k} d \mathbf{x}}{\int_{\Omega} \phi_{k} d \mathbf{x}} \text { for } k=1, \ldots, K .
$$

Variation of energy (2.2) with respect to $\phi$ yields the following gradient descent equation (the modified vector-valued AC equation):

$$
\frac{\partial \phi}{\partial t}=-\frac{\mathbf{f}(\phi)}{\epsilon^{2}}+\Delta \phi-\lambda \mathbf{G}(\phi)+\beta(\phi) \mathbf{1}
$$

where $\mathbf{G}(\phi)=\left(\phi_{1}\left(I-c_{1}\right)^{2}, \ldots, \phi_{K}\left(I-c_{K}\right)^{2}\right)$ and

$$
\beta(\phi)=\frac{1}{K} \sum_{k=1}^{K}\left[\frac{f\left(\phi_{k}\right)}{\epsilon^{2}}+\lambda \phi_{k}\left(I-c_{k}\right)^{2}\right]
$$

is a variable Lagrangian multiplier. We note that in [35] a scalar phase-field with $(K-1)$ level was used to represent $K$ phases and a periodic quartic polynomial was used as a potential. In our model, a vector-valued phase-field with $K$ components is used to represent $K$ phases and a double well potential is used, which is typically used in the Cahn-Hilliard and the Allen-Cahn equations.

\section{AN OPERATOR SPLITTING METHOD FOR VECTOR-VALUED AC EQUATIONS}

In this section, we describe an operator splitting method for solving the modified vectorvalued AC equation (2.3). Since $\phi_{K}=1-\phi_{1}-\phi_{2}-\ldots-\phi_{K-1}$, we only need to solve equations with $\phi_{1}, \phi_{2}, \ldots, \phi_{K-1}$, and $\phi=\left(\phi_{1}, \phi_{2}, \ldots, \phi_{K-1}\right)$ can be redefined with only $K-1$ variables. Similarly we redefine $\mathbf{f}(\phi), \mathbf{G}(\phi)$, and $\mathbf{1}$ to $\mathbf{f}(\phi)=\left(\frac{\partial F}{\partial \phi_{1}}, \ldots, \frac{\partial F}{\partial \phi_{K-1}}\right)$, $\mathbf{G}(\phi)=\left(\phi_{1}\left(I-c_{1}\right)^{2}, \ldots, \phi_{K-1}\left(I-c_{K-1}\right)^{2}\right)$, and $\mathbf{1}=(1, \ldots, 1) \in \mathbb{R}^{K-1}$.

Let $\Omega=(a, b) \times(c, d)$ be the computational domain in $2 \mathrm{D}, N_{x}$ and $N_{y}$ be positive even integers, $h=(b-a) / N_{x}=(d-c) / N_{y}$ be a uniform mesh size, and $\Omega_{h}=\left\{\left(x_{i}, y_{j}\right): x_{i}=\right.$ $\left.(i-0.5) h, y_{j}=(j-0.5) h, 1 \leq i \leq N_{x}, 1 \leq j \leq N_{y}\right\}$ be the set of cell-centers. Let $\phi_{i j}^{n}$ be the approximations of $\phi\left(x_{i}, y_{j}, n \Delta t\right)$, where $\Delta t=T / N_{t}$ is the time step, $T$ is the final time, and $N_{t}$ is the total number of time steps. We define the discrete differentiation operators by

$$
D_{x} \phi_{i+\frac{1}{2}, j}=\frac{1}{h}\left(\phi_{i+1, j}-\phi_{i j}\right) \quad \text { and } \quad D_{y} \phi_{i, j+\frac{1}{2}}=\frac{1}{h}\left(\phi_{i, j+1}-\phi_{i j}\right) .
$$


and the discrete Laplacian by

$$
\Delta_{d} \boldsymbol{\phi}_{i j}=\frac{1}{h}\left(D_{x} \boldsymbol{\phi}_{i+\frac{1}{2}, j}-D_{x} \boldsymbol{\phi}_{i-\frac{1}{2}, j}+D_{y} \boldsymbol{\phi}_{i, j+\frac{1}{2}}-D_{y} \boldsymbol{\phi}_{i, j-\frac{1}{2}}\right) .
$$

We discretize (2.3) in time by an operator splitting algorithm:

$$
\begin{aligned}
\frac{\boldsymbol{\phi}_{i j}^{*}-\boldsymbol{\phi}_{i j}^{n}}{\Delta t} & =\Delta_{d} \boldsymbol{\phi}_{i j}^{*}-\lambda \mathbf{G}\left(\boldsymbol{\phi}_{i j}^{n}\right)+\beta\left(\boldsymbol{\phi}_{i j}^{n}\right) \mathbf{1}, \\
\frac{\boldsymbol{\phi}_{i j}^{n+1}-\boldsymbol{\phi}_{i j}^{*}}{\Delta t} & =-\frac{\mathbf{f}\left(\boldsymbol{\phi}_{i j}^{n+1}\right)}{\epsilon^{2}},
\end{aligned}
$$

where $c_{k}^{n}$ is defined as follows:

$$
c_{k}^{n}=\sum_{i=1}^{N_{x}} \sum_{j=1}^{N_{y}} I_{i j}\left(\phi_{k}\right)_{i j}^{n} / \sum_{i=1}^{N_{x}} \sum_{j=1}^{N_{y}}\left(\phi_{k}\right)_{i j}^{n}
$$

for $k=1, \ldots, K-1$.

Equation (3.1) is an implicit Euler's method for $\phi_{t}=\Delta \phi-\lambda \mathbf{G}(\phi)+\beta(\phi) \mathbf{1}$ with an initial condition $\phi^{n}$. The resulting implicit discrete system of equations can be solved by a fast solver, such as a multigrid method $[49,50]$. Also, a pointwise Gauss-Seidel relaxation scheme is used as the smoother in the multigrid method. Equation (3.2) can be considered as an approximation of the equation

$$
\phi_{t}=-\frac{\mathbf{f}(\phi)}{\epsilon^{2}}
$$

by an implicit Euler's method with an initial condition $\phi^{*}$. We can solve (3.3) semi-analytically using a closed-form solution. The solution is given as follows:

$$
\left(\phi_{k}\right)_{i j}^{n+1}=0.5+\frac{\left(\phi_{k}\right)_{i j}^{*}-0.5}{\sqrt{e^{-\frac{\Delta t}{2 \epsilon^{2}}}+\left(2\left(\phi_{k}\right)_{i j}^{*}-1\right)^{2}\left(1-e^{-\frac{\Delta t}{2 \epsilon^{2}}}\right)}}
$$

for $k=1, \ldots, K-1$. The numerical algorithm is shown schematically in Figure 1 .

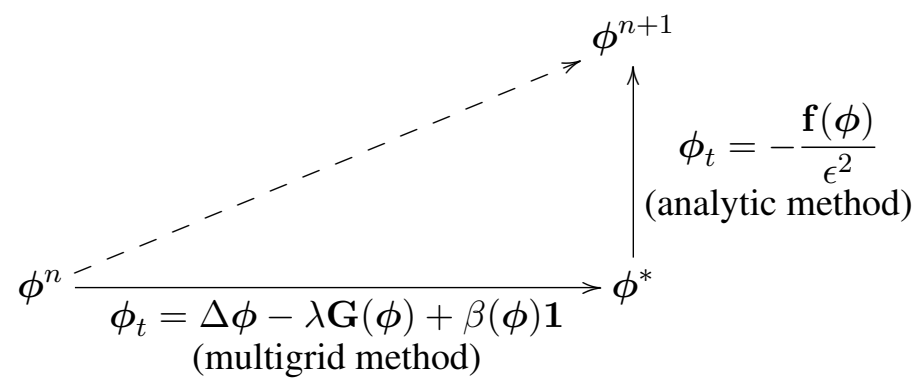

FIGURE 1. Schemetic diagram of operator splitting method. 
In (3.1), the variable Lagrange multiplier $\beta(\phi)$ is determined by the solutions at time level $n$. By treating $\beta(\phi)$ explicitly, there is no relation between the solutions at time level $*$. Thus the modified vector-valued $\mathrm{AC}$ equation can be solved in a decoupled way,

$$
\begin{aligned}
\frac{\left(\phi_{k}\right)_{i j}^{*}-\left(\phi_{k}\right)_{i j}^{n}}{\Delta t} & =\Delta_{d}\left(\phi_{k}\right)_{i j}^{*}-\lambda\left(\phi_{k}\right)_{i j}^{n}\left(I_{i j}-c_{k}^{n}\right)^{2}+\beta\left(\phi_{i j}^{n}\right), \\
\left(\phi_{k}\right)_{i j}^{n+1} & =0.5+\frac{\left(\phi_{k}\right)_{i j}^{*}-0.5}{\sqrt{e^{-\frac{\Delta t}{2 \epsilon^{2}}}+\left(2\left(\phi_{k}\right)_{i j}^{*}-1\right)^{2}\left(1-e^{-\frac{\Delta t}{2 \epsilon^{2}}}\right.}}
\end{aligned}
$$

for $k=1, \ldots, K-1$. This means that we only solve the modified scalar AC equation $K-1$ times to solve the modified vector-valued AC equation.

\section{NUMERICAL EXPERIMENTS FOR IMAGE SEGMENTATION}

In this section, we demonstrate numerical efficiency and stability of the operator splitting method (3.4)-(3.5) described in the previous section for solving the modified vector-valued AC equation (2.3). A steady state solution of (2.3) is controlled by two tuning parameters: $\epsilon$ for image sharpness (interface thickness) and $\lambda$ for image fidelity (closeness to the original image). For given fixed value of $\lambda$, interface width parameter $\epsilon$ plays an important role of the final image sharpness. We want to set the phase-field variable varies across an interfacial region from 0.05 to 0.95 over a distance of about $4 \sqrt{2} \epsilon \tanh ^{-1}(0.9)$. Therefore, if we want this value to be approximately $m$ grid points, the $\epsilon$ value needs to be taken as $\epsilon_{m}=h m /\left(4 \sqrt{2} \tanh ^{-1}(0.9)\right)$. In this paper, we chose various $\epsilon$ values which are suitable for each problem, however, different $\epsilon$ values make little differences in the results. In all the numerical experiments, a given image $I$ is normalized as $I=\frac{I-I_{\min }}{I_{\max }-I_{\min }}$, where $I_{\max }$ and $I_{\min }$ are the maximum and minimum values of the given image, respectively. Note that we use the same computational domain $\Omega=(0,1) \times$ $(0,1)$ for the sake of simplicity.

We provide 3 examples in this section: one for testing computational speed of the numerical algorithm with multiple phase components, one for checking numerical stability of the multiphase image segmentation method with noisy data, and one for demonstrating suitability of the algorithm for real world images. We give the CPU time in seconds for our calculations, performed on a workstation with a $3.2 \mathrm{GHz}$ Intel Core i5 CPU and 4 GB of RAM.

\section{Example 1. (Computational efficiency with multi-components) Synthetic image}

The first example, Figure 2, shows a complex synthetic image taken from [48] that contains several generic visual structures, such as an internal hole, occlusion and stacked objects, Tjunctions, singular junctions. The parameter values are chosen as $h=1 / 256, \Delta t=5 \mathrm{E}-6$, $\epsilon=\epsilon_{5}$, and $\lambda=10$. For segmentation, it took only 2 iterations and 0.594 seconds, while the previous method in [48] took 18 iterations and 37.109 seconds. The results with $K=5$ phasefield components in Figure 2 show that our method can capture multiple objects simultaneously for multiple geometries.

One of the drawbacks for any method based on the AC equation is that the number $K$ of components of $\phi$ must presently be specified from the start, and we did not consider the 


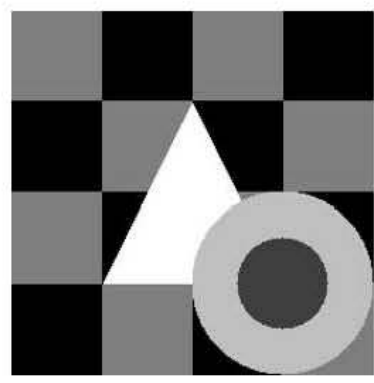

Original image

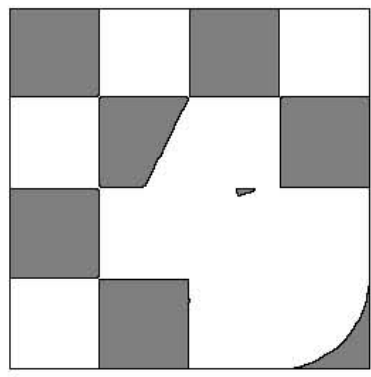

$\phi_{3}, c_{3}=0.4977$

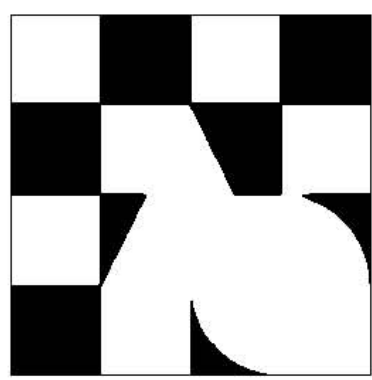

$\phi_{1}, c_{1}=0.0243$

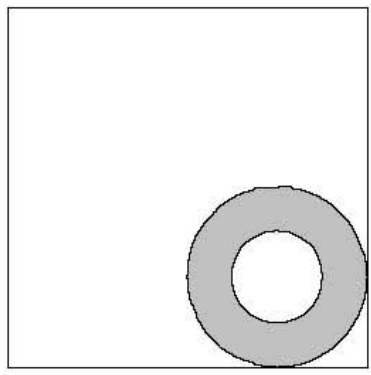

$\phi_{4}, c_{4}=0.7521$

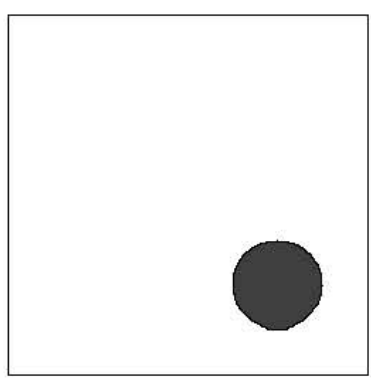

$\phi_{2}, c_{2}=0.2490$

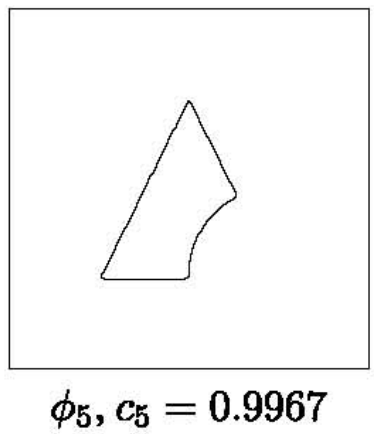

FIGURE 2. Original image $I$ with multiple objects of size $256 \times 256$ and steady state $(n \geq 2)$ solutions, $\phi_{k}, c_{k}, k=1, \ldots, 5$ for $\epsilon=\epsilon_{5}$ and $\lambda=10$.
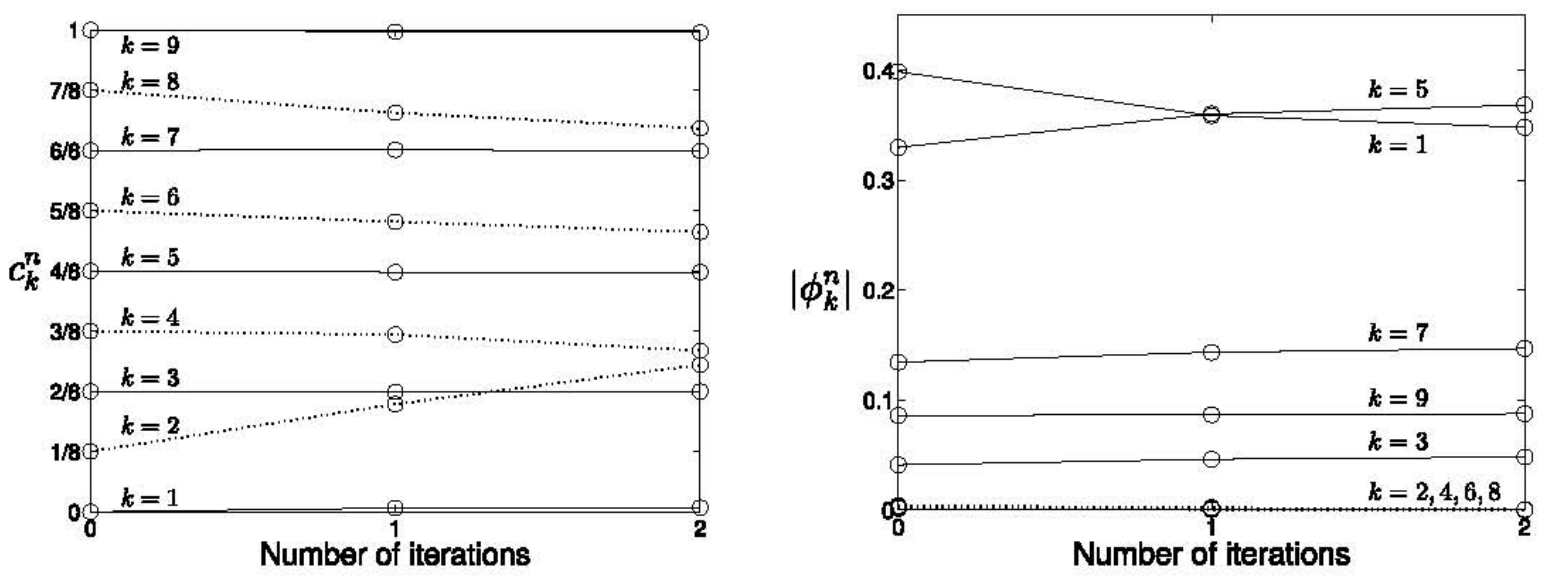

FIGURE 3. Segmentation of the image in Figure 2 with $K=9$.

feasibility or implications of adding or removing components during the computation. Thus, to segment a multiphase image successfully, we need a sufficient number of phase-field variables. Figure 3 shows the convergence result for the same image shown in the previous figure with $K=9$ phase-field components which is larger than the require image levels, $K=5$. The algorithm successfully finds the 5 components (marked in solid lines in the figure) and the rest 
4 components (with zero size $\left|\phi_{k}^{n}\right| \approx 0$, marked with dotted lines) do not disturb convergence of the iterations.

In general, the computing time increases nonlinearly with the number of phase-field variables $K$. However, with our method, the computing time increases linearly with the number of phase-field variables, since we can solve the modified vector-valued AC equation in a decoupled way. In order to show the efficiency of our method, we consider the same problem in Figure 2 with $K=5,9,13,17$, and 21. Figure 4 shows the computing time for the segmentation as a function of phase-variables $K$. The result suggests that the convergence rate of computing time is linear with respect to number of components, $T_{\mathrm{CPU}} \approx C_{T}(K-1)$, where $C_{T}=0.166$.

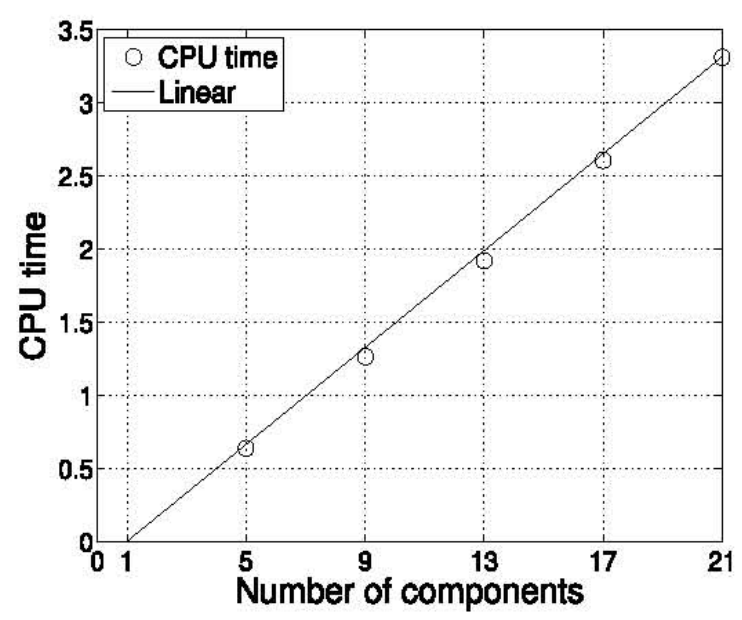

FIGURE 4. CPU time versus number of components.

To show the effect of interface width parameter $\epsilon$ on the segmentation result, we consider the same problem in Figure 2 with $\epsilon=\epsilon_{3}$. Figure 5 shows $\phi_{k}^{n}, k=1, \ldots, 5$ after $n=2$ iterations. In contrast to the result in Figure 2, $\phi_{1}$ in Figure 5 still contains the noise of the original image. In the case of $\epsilon=\epsilon_{3}$, the noise is completely eliminated after $n=4$ iterations. From this result, we observe that the segmentation result depends on the interface width parameter $\epsilon$.

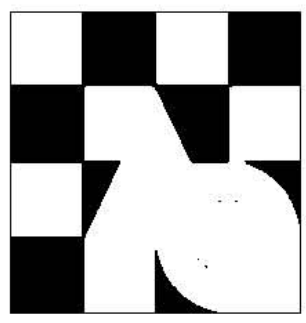

$\phi_{1}, c_{1}=0.0198$

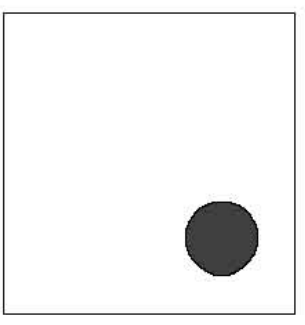

$\phi_{2}, c_{2}=0.2484$

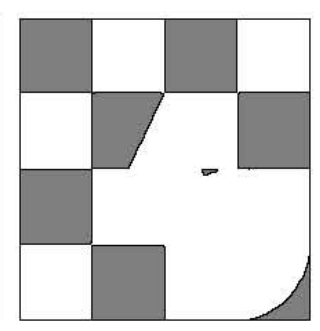

$\phi_{3}, c_{3}=0.4978$

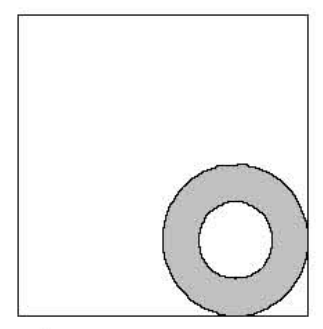

$\phi_{4}, c_{4}=0.7524$

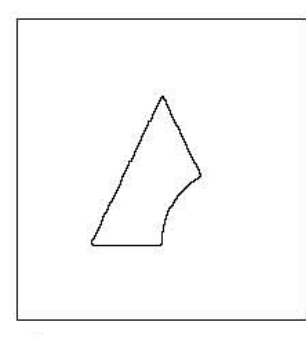

$\phi_{5}, c_{5}=0.9969$

FIGURE 5. Solutions after 2 iterations, $\phi_{k}, c_{k}, k=1, \ldots, 5$ for $\epsilon=\epsilon_{3}$ and $\lambda=10$. 


\section{Example 2. (Stability for the phase-field component number $K$ ) Synthetic noisy image}

To show the effectiveness and stability of our method for over-sampled phase-field components, we choose a typical example from [47]. A noisy synthetic image contains 4 stars on 4 different backgrounds (see Figure 6). The star in the upper left corner belongs to same phase as the background in the upper right corner. Further, the star in the upper right corner belongs to the same phase as the background in the lower left corner, and the star in this corner belongs to the same phase as the background in the lower right corner. The parameter values are chosen as $h=1 / 256, \Delta t=4 \mathrm{E}-6, \epsilon=\epsilon_{3}$, and $\lambda=10$. As we can see in Figure 6, our method gives clear results with the five phase-field variables after 10 iterations and 2.97 seconds.

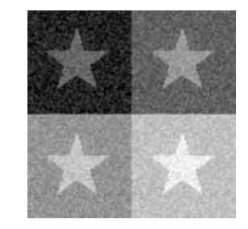

Original image

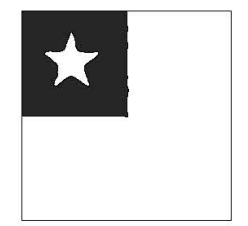

$\phi_{1}$

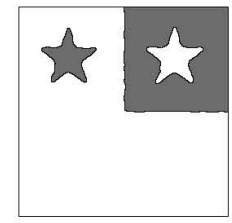

$\phi_{2}$

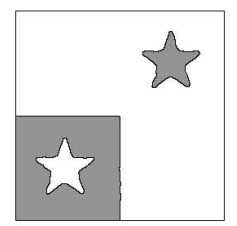

$\phi_{3}$

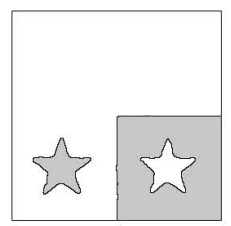

$\phi_{4}$

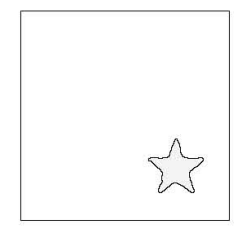

$\phi_{5}$

FIGURE 6. Original image $I$ of size $256 \times 256$ with noise and segmented images for $K=5, \epsilon=\epsilon_{3}$, and $\lambda=10$.

We now increase the number of components $K$ bigger than 5 to check the stability of the numerical algorithm with respect to $K$. Figure 7 shows $\phi_{k}^{n}, k=1, \ldots, 6$ after $n=10$ iterations. Although the second component is still not well separated from the third after 10 iterations, we can observe that the resulting phase-field functions are converging even with over-sampled phase-field components.

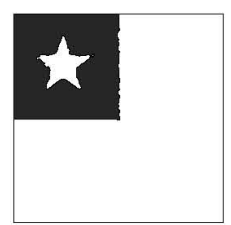

$\phi_{1}$

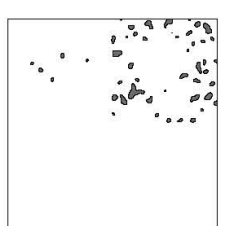

$\phi_{2}$

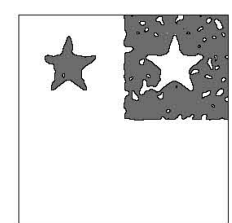

$\phi_{3}$

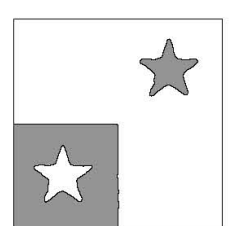

$\phi_{4}$

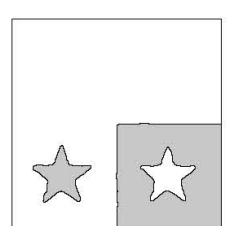

$\phi_{5}$

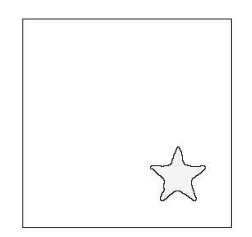

$\phi_{6}$

FIGURE 7. Segmented images for $K=6, \epsilon=\epsilon_{3}$, and $\lambda=10$.

We further increase the number of components to $K=9$. Figure 8 shows the size of phasefield function and average image intensity $\left|\phi_{k}^{n}\right|, c_{k}^{n}$ for $k=1, \ldots, K$ as functions of iteration. And also phase-field functions after 10 iterations $\phi_{k}^{10}$ are visualized in the figure. We stopped the algorithm after 10 iterations just for the demonstration and some of the components with residual such as $k=2,4,6$ are still vanishing. This example experimentally suggests that the algorithm is numerically stable when initial number of components is over-sampled. 


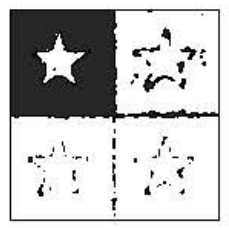

$\phi_{1}$

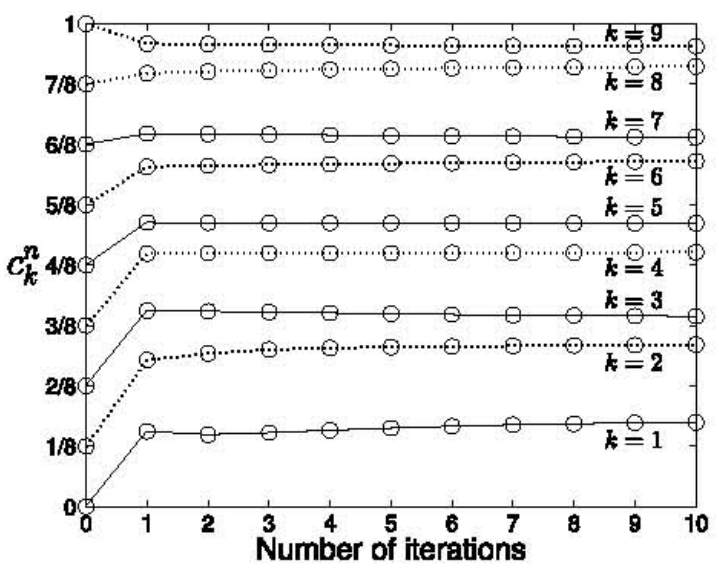

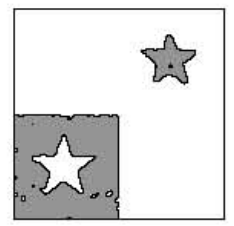

$\phi_{5}$

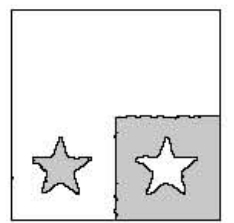

$\phi_{6}+\phi_{7}$

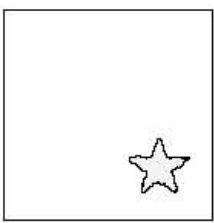

$\phi_{8}+\phi_{9}$

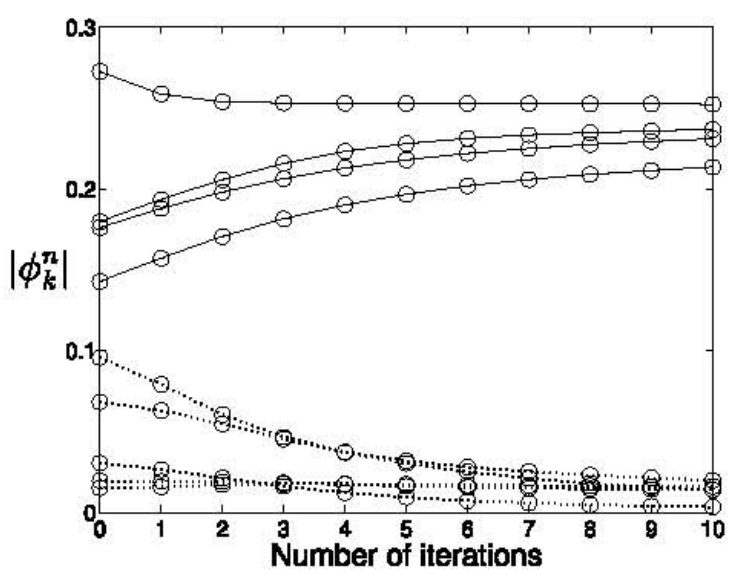

FIGURE 8. Convergence of the iteration and the segmented images for $K=9$, $\epsilon=\epsilon_{3}$, and $\lambda=10 .\left|\phi_{10}^{10}\right|>\left|\phi_{5}^{10}\right|>\left|\phi_{7}^{10}\right|>\left|\phi_{3}^{10}\right|$ (marked with solid lines) and $\left|\phi_{2}^{10}\right|>\left|\phi_{9}^{10}\right|>\left|\phi_{8}^{10}\right|>\left|\phi_{6}^{10}\right|>\left|\phi_{4}^{10}\right|$ (marked with dotted lines).

Example 3. (Segmentation results for real life application) landscape and brain images

Figure 9 shows the application of our method to a real landscape image. This example is taken from [35]. The parameter values are chosen as $h=1 / 256, \Delta t=4 \mathrm{E}-6, \epsilon=\epsilon_{3}$, and $\lambda=10$. Figure 9 shows steady state segmentation results with $K=6$ after $n=5$ iterations (taken 1.938 seconds). We observe that different objects in the real landscape image are correctly detected and the image is quickly segmented.

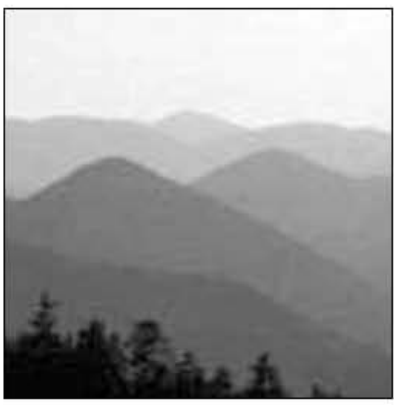

Original image

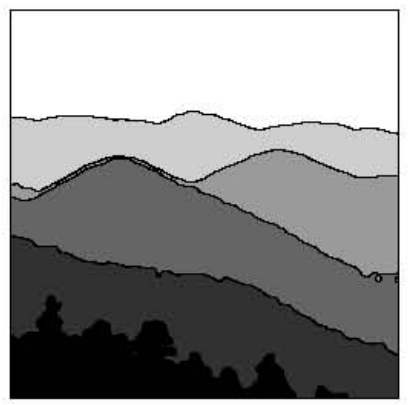

(a)

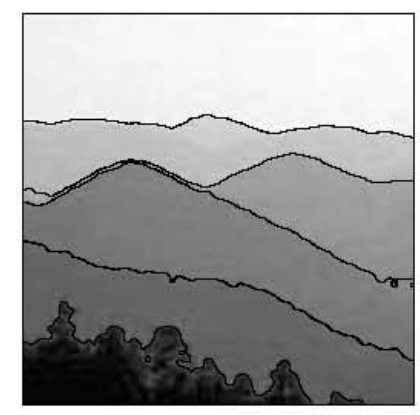

(b)

FIGURE 9. Segmentation of a real landscape image of size $256 \times 256$ for $\epsilon=\epsilon_{3}$ and $\lambda=10$. (a) 6 segmented regions filled with color intensity $c_{k}, k=$ $1, \ldots, 6(\mathrm{~b})$ the same 6 regions overlayed with the original image. 
Figure 10 shows the application of our method to a MR brain image. This example is taken from [48]. The parameter values are chosen as $h=1 / 256, \Delta t=5 \mathrm{E}-6, \epsilon=\epsilon_{2}$, and $\lambda=10$. Even though the intensities fluctuate severely and the boundaries are complex, our method separates satisfactorily the major different phases. Shown on top of the original image are the three small patches that are in practice easily supervised by a radiologist. For segmentation with $K=3$, it took only 7 iterations and 1.156 seconds.

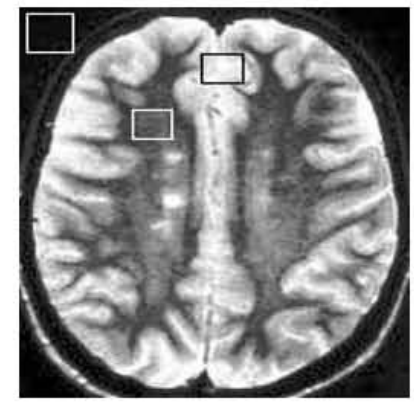

Original image

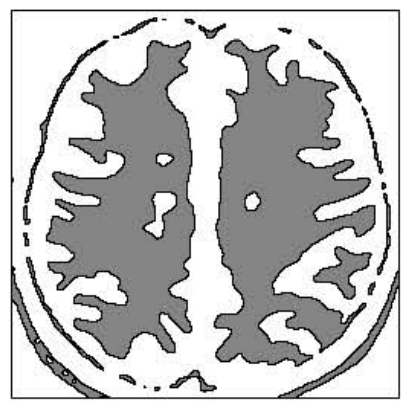

$\phi_{2}, c_{2}=0.5205$

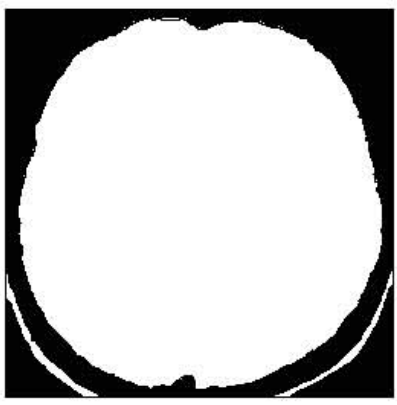

$\phi_{1}, c_{1}=0.0078$

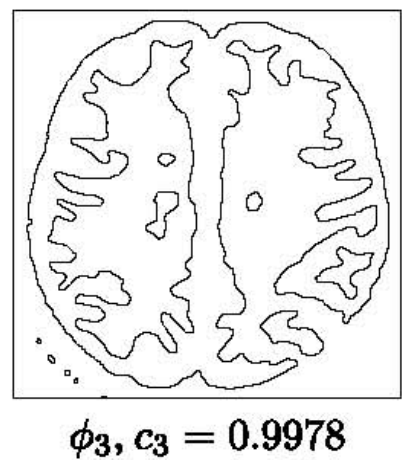

FIGURE 10. Original MR brain image of size $256 \times 256$ and steady state $(n \geq 7)$ solutions, $\phi_{k}, c_{k}, k=1,2,3$ for $\epsilon=\epsilon_{2}$ and $\lambda=10$.

\section{ConClusions}

In this paper, we presented an efficient numerical method for multiphase image segmentation using a multiphase-field model. The method combines the vector-valued Allen-Cahn phase-field equation with initial data fitting terms containing prescribed interface width and fidelity constants. An efficient numerical solution was achieved using the recently developed hybrid operator splitting method for the vector-valued Allen-Cahn phase-field equation. We split the modified vector-valued Allen-Cahn equation into a nonlinear equation and a linear diffusion equation with a source term (the initial data fitting terms and the constraint terms). The linear diffusion equation was discretized using an implicit scheme and the resulting implicit discrete system of equations was solved by a multigrid method. The nonlinear equation was solved semi-analytically using a closed-form solution. And by treating the source term of the 
linear diffusion equation explicitly, we solved the modified vector-valued Allen-Cahn equation in a decoupled way. By decoupling the governing equation, we could speed up the segmentation process with multiple phases. We performed some characteristic numerical experiments for multiphase image segmentation. The method is computationally linear algorithm with respect to the number of phase-field components and numerically stable even when the image is over-sampled with phase-field components much more than the required number. These two characteristics make the algorithm to be a nice tool for real world image segmentation with noisy and complicated structures.

\section{REFERENCES}

[1] S. M. Allen and J. W. Cahn, A microscopic theory for antiphase boundary motion and its application to antiphase domain coarsening, Acta Metallurgica, 27 (1979), 1085-1095.

[2] A. A. Wheeler, W. J. Boettinger and G. B. McFadden, Phase-field model for isothermal phase transitions in binary alloys, Physical Review A, 45 (1992), 7424-7439.

[3] M. Cheng and J. A. Warren, An efficient algorithm for solving the phase field crystal model, Journal of Computational Physics, 227 (2008), 6241-6248.

[4] Y. Li, H. G. Lee and J. Kim, A fast, robust, and accurate operator splitting method for phase-field simulations of crystal growth, Journal of Crystal Growth, 321 (2011), 176-182.

[5] Y. Li, D. Lee, H. G. Lee, D. Jeong, C. Lee, D. Yang and J. Kim, A robust and accurate phase-field simulation of snow crystal growth, Journal of the Korean Society for Industrial and Applied Mathematics, 16 (2012), $15-29$.

[6] L.-Q. Chen and W. Yang, Computer simulation of the domain dynamics of a quenched system with a large number of nonconserved order parameters: The grain-growth kinetics, Physical Review B, 50 (1994), 1575215756.

[7] I. Steinbach, F. Pezzolla, B. Nestler, M. Seeßelberg, R. Prieler, G. J. Schmitz and J. L. L. Rezende, A phase field concept for multiphase systems, Physica D, 94 (1996), 135-147.

[8] D. Fan, C. Geng and L.-Q. Chen, Computer simulation of topological evolution in 2-D grain growth using a continuum diffuse-interface field model, Acta Materialia, 45 (1997), 1115-1126.

[9] M. T. Lusk, A phase-field paradigm for grain growth and recrystallization, Proceedings of the Royal Society of London A, 455 (1999), 677-700.

[10] R. Kobayashi, J. A. Warren and W. C. Carter, A continuum model of grain boundaries, Physica D, 140 (2000), 141-150.

[11] M. Beneš, V. Chalupecký and K. Mikula, Geometrical image segmentation by the Allen-Cahn equation, Applied Numerical Mathematics, 51 (2004), 187-205.

[12] J. A. Dobrosotskaya and A. L. Bertozzi, A wavelet-Laplace variational technique for image deconvolution and inpainting, IEEE Transactions on Image Processing, 17 (2008), 657-663.

[13] L. C. Evans, H. M. Soner and P. E. Souganidis, Phase transitions and generalized motion by mean curvature, Communications on Pure and Applied Mathematics, 45 (1992), 1097-1123.

[14] T. Ilmanen, Convergence of the Allen-Cahn equation to Brakke's motion by mean curvature, Journal of Differential Geometry, 38 (1993), 417-461.

[15] M. Katsoulakis, G. T. Kossioris and F. Reitich, Generalized motion by mean curvature with Neumann conditions and the Allen-Cahn model for phase transitions, The Journal of Geometric Analysis , 5 (1995), 255-279.

[16] L. Q. Chen and J. Shen, Applications of semi-implicit Fourier-spectral method to phase field equations, Computer Physics Communications, 108 (1998), 147-158.

[17] M. Beneš and K. Mikula, Simulation of anisotropic motion by mean curvature-comparison of phase-field and sharp-interface approaches, Acta Mathematica Universitatis Comenianae, 67 (1998), 17-42. 
[18] X. Feng and A. Prohl, Numerical analysis of the Allen-Cahn equation and approximation for mean curvature flows, Numerische Mathematik, 94 (2003), 33-65.

[19] T. Ohtsuka, Motion of interfaces by an Allen-Cahn type equation with multiple-well potentials, Asymptotic Analysis, 56 (2008), 87-123.

[20] X. Yang, J. J. Feng, C. Liu and J. Shen, Numerical simulations of jet pinching-off and drop formation using an energetic variational phase-field method, Journal of Computational Physics, 218 (2006), 417-428.

[21] Q. Du, C. Liu and X. Wang, A phase field approach in the numerical study of the elastic bending energy for vesicle membranes, Journal of Computational Physics, 198 (2004), 450-468.

[22] J. A. Sethian and J. Strain, Crystal growth and dendritic solidification, Journal of Computational Physics, 98 (1992), 231-253.

[23] S. Li, J. S. Lowengrub and P. H. Leo, Nonlinear morphological control of growing crystals, Physica D, 208 (2005), 209-219.

[24] D. Li, R. Li and P. Zhang, A cellular automaton technique for modelling of a binary dendritic growth with convection, Applied Mathematical Modelling, 31 (2007), 971-982.

[25] H. Yin and S. D. Felicelli, A cellular automaton model for dendrite growth in magnesium alloy AZ91, Modelling and Simulation in Materials Science and Engineering, 17 (2009), 075011.

[26] D. Juric and G. Tryggvason, A front-tracking method for dendritic solidification, Journal of Computational Physics, 123 (1996), 127-148.

[27] D. Stafford, M. J. Ward and B. Wetton, The dynamics of drops and attached interfaces for the constrained Allen-Cahn equation, European Journal of Applied Mathematics, 12 (2001), 1-24.

[28] S. Chen, B. Merriman, S. Osher and P. Smereka, A simple level set method for solving Stefan problems, Journal of Computational Physics, 135 (1997), 8-29.

[29] L.-L. Wang and Y. Gu, Efficient dual algorithms for image segmentation using TV-Allen-Cahn type models, Communications in Computational Physics, 9 (2011), 859-877.

[30] Z. Xu, H. Huang, X. Li and P. Meakin, Phase field and level set methods for modeling solute precipitation and/or dissolution, Computer Physics Communications, 183 (2012), 15-19.

[31] A. E. Lobkovsky and J. A. Warren, Phase-field model of crystal grains, Journal of Crystal Growth, 225 (2001), 282-288.

[32] B. Nestler and A. A. Wheeler, Phase-field modeling of multi-phase solidification, Computer Physics Communications, 147 (2002), 230-233.

[33] J.-W. Choi, H. G. Lee, D. Jeong and J. Kim, An unconditionally gradient stable numerical method for solving the Allen-Cahn equation, Physica A, 388 (2009), 1791-1803.

[34] Y. Li, H. G. Lee, D. Jeong and J. Kim, An unconditionally stable hybrid numerical method for solving the Allen-Cahn equation, Computers and Mathematics with Applications, 60 (2010), 1591-1606.

[35] Y. Li and J. Kim, Multiphase image segmentation using a phase-field model, Computers \& Mathematics with Applications, 62 (2011), 737-745.

[36] H. Garcke, B. Nestler and B. Stoth, On anisotropic order parameter models for multi-phase systems and their sharp interface limits, Physica D, 115 (1998), 87-108.

[37] H. Garcke, B. Nestler and B. Stinner, A diffuse interface model for alloys with multiple components and phases, SIAM Journal on Applied Mathematics, 64 (2004), 775-799.

[38] H. Garcke and V. Styles, Bi-directional diffusion induced grain boundary motion with triple junctions, Interfaces and Free Boundaries, 6 (2004), 271-294.

[39] B. Nestler, H. Garcke and B. Stinner, Multicomponent alloy solidification: Phase-field modeling and simulations, Physical Review E, 71 (2005), 041609.

[40] N. Moelans, B. Blanpain and P. Wollants, A phase field model for the simulation of grain growth in materials containing finely dispersed incoherent second-phase particles, Acta Materialia, 53 (2005), 1771-1781.

[41] R. Kornhuber and R. Krause, Robust multigrid methods for vector-valued Allen-Cahn equations with logarithmic free energy, Computing and Visualization in Science, 9 (2006), 103-116. 
[42] D. A. Kay and A. Tomasi, Color image segmentation by the vector-valued Allen-Cahn phase-field model: a multigrid solution, IEEE Transactions on Image Processing, 18 (2009), 2330-2339.

[43] L. Vanherpe, F. Wendler, B. Nestler and S. Vandewalle, A multigrid solver for phase field simulation of microstructure evolution, Mathematics and Computers in Simulation, 80 (2010), 1438-1448.

[44] H. G. Lee and J. Kim, An efficient and accurate numerical algorithm for the vector-valued Allen-Cahn equations, Computer Physics Communications, 183 (2012), 2107-2115.

[45] L. A. Vese and T. F. Chan, A multiphase level set framework for image segmentation using the Mumford and Shah model, International Journal of Computer Vision, 50 (2002), 271-293.

[46] C. Samson, L. Blanc-Féraud, G. Aubert and J. Zerubia, A level set model for image classification, International Journal of Computer Vision, 40 (2000), 187-197.

[47] J. Lie, M. Lysaker and X.-C. Tai, A variant of the level set method and applications to image segmentation, Mathematics of computation, 75 (2006), 1155-1174.

[48] Y. M. Jung, S. H. Kang and J. Shen, Multiphase image segmentation via Modica-Mortola phase transition, SIAM Journal on Applied Mathematics, 67 (2007), 1213-1232.

[49] W. L. Briggs, A Multigrid Tutorial, SIAM, Philadelphia, PA, 1987.

[50] U. Trottenberg, C. Oosterlee and A. Schüller, Multigrid, Academic Press, London, 2001

[51] D. Mumford and J. Shah, Optimal approximations by piecewise smooth functions and associated variational problems, Communications on Pure and Applied Mathematics, 42 (1989), 577-685.

[52] S. C. Zhu and A. Yuille, Region competition: unifying snakes, region growing, and Bayes/MDL for multiband image segmentation, IEEE Transactions on Pattern Analysis and Machine Intelligence, 18 (1996), 884-900.

[53] M. Kass, A. Witkin and D. Terzopoulos, Snakes: Active contour models, International Journal of Computer Vision, 1 (1988), 321-331.

[54] N. Paragios and R. Deriche, Geodesic active regions for supervised texture segmentation, The Proceedings of the Seventh IEEE International Conference on Computer Vision, Corfu, Greece 1999.

[55] T. F. Chan and L. A. Vese, Active contours without edges, IEEE Transactions on Image Processing, 10 (2001), 266-277. 